\title{
Estudo de equivalência farmacêutica entre comprimidos de furosemida similares e genéricos
}

\author{
Pharmaceutical equivalence study between similar and generic \\ furosemide pills
}

Recebido em: 26/09/2019 Aceito em: $02 / 03 / 2020$
Murilo Neth de SOUSA ${ }^{1}$; Thalita Prates da SILVA ${ }^{2}$; Emilly Isabelli dos Santos TEODORO²; Mariana Aparecida LOPES-ORTIZ ${ }^{1}$; João Carlos PALAZZO DE MELLO' ${ }^{2}$ : Daniela Cristina de Medeiros ARAÚJO' ${ }^{I}$ Centro Universitário Ingá - Uningá. Rod. PR 317, 6114 Parque Industrial 200, CEP 87035-510. Maringá, PR, Brasil. ${ }^{2}$ Universidade Estadual de Maringá. Av. Colombo, 5790, Zona 7, CEP 87020-900. Maringá, PR, Brasil. E-mail:danielamedeiros@hotmail.com

\section{ABSTRACT}

Furosemide is a medicine widely used in hypertensive therapy, congestive heart failure, oedema of cardiac, renal and hepatic origin. Because it is a loop diuretic, due to its role in the thick segment of the Henle loop, it is considered very potent for these diseases. Pharmaceutical equivalence aims to promote comparative quality tests between the reference drug and the drug to be tested. Test and reference medicinal products must present the same concentrations, pharmaceutical forms, physical and physicochemical characteristics. In this study, the drug furosemide $40 \mathrm{mg}$ was used, where two generic brands were tested (G1 and G2), and two similar ones (S1 and S2) comparing them with the reference (R). Regarding weight variation, friability, disintegration, hardness, dissolution, dosing and the total number of mesophilic microorganisms, it can be said that the drugs are pharmaceutical equivalents.

Keywords: pharmaceutical equivalence; furosemide; generic; similar drug.

\section{RESUMO}

A furosemida é um medicamento muito utilizado em terapia hipertensiva, insuficiência cardíaca congestiva, edema de origem cardíaca, renal e hepática. Por se tratar de um diurético de alça, em relação à sua atuação no segmento espesso da alça de Henle, é considerado muito potente para essas doenças. A equivalência farmacêutica tem como objetivo promover testes comparativos de qualidade entre o medicamento referência e o medicamento que será testado. Os medicamentos-teste e o referência devem apresentar as mesmas concentrações, formas farmacêuticas, características físicas e físico-químicas. Neste trabalho, foi utilizado o medicamento furosemida $40 \mathrm{mg}$, onde foram testadas duas marcas de genéricos (G1 e G2), e duas similares (S1 e S2) comparando-as com o referência (R). Quanto à variação de peso, friabilidade, desintegração, dureza, dissolução, doseamento e número total de microorganismos mesófilos, os medicamentos foram considerados equivalentes farmacêuticos.

Palavras-chave: equivalência farmacêutica; furosemida; genérico; medicamento similar. 


\section{INTRODUÇÃO}

Estima-se que a cada ano ocorrem nas Américas cerca de 1,6 milhões de mortes por doenças cardiovasculares relacionadas à hipertensão arterial. Desses óbitos, cerca de meio milhão ocorrem entre pessoas com menos de 70 anos, segundo a Organização Panamericana de Assistência à Saúde (OPAS), sendo que um dos maiores desafios de saúde pública é a hipertensão. A furosemida, por se tratar de um coadjuvante no tratamento dessa doença, vem sendo cada vez mais estudada (1-2).

Para facilitar seu manuseio, melhorar a aparência física das formulações, estabilidade e desintegração, além do fármaco ativo, são adicionados excipientes inertes na fabricação de comprimidos e cápsulas. São necessários muitos cuidados em relação a essas substâncias, pois embora sejam chamadas de inertes, podem interferir na liberação e posterior absorção do fármaco. Desse modo, são indispensáveis testes para garantir qualidade, segurança e eficácia do medicamento (3).

A furosemida é um medicamento muito utilizado na terapia hipertensiva, insuficiência cardíaca congestiva, edema de origem cardíaca, renal e hepática. Por se tratar de um diurético de alça, em relação a sua atuação no segmento espesso da alça de Henle, é considerado muito potente para essas doenças. É um medicamento pertence à classe IV do sistema de classificação biofarmacêutica, por possuir baixa permeabilidade e baixa solubilidade (4-7).

Seu mecanismo de ação se baseia em bloquear o sistema cotransportador de $\mathrm{Na}^{+} \mathrm{K}^{+} 2 \mathrm{Cl}$ localizado no ramo ascendente da alça de Henle. Portanto, a eficácia do medicamento é dependente do alcance do lúmen tubular através de um mecanismo de transporte aniônico. Neste segmento da alça, irá ocorrer uma reabsorção de cloreto de sódio, caracterizando sua ação diurética (8).

Diante da Resolução da Diretoria Colegiada da Agência Nacional de Vigilância Sanitária (Anvisa) (RDC) no 17 de 16 de abril de 2010 que dispõe sobre "Boas Práticas de Fabricação de Medicamentos", o controle da qualidade é o conjunto de técnicas e atividades operacionais utilizadas para monitorar o cumprimento dos requisitos da qualidade especificados (9).
Atrelado ao controle da qualidade está a equivalência farmacêutica. A equivalência farmacêutica tem como objetivo promover testes comparativos de qualidade entre o medicamento referência e o(s) medicamento(s) teste. $\mathrm{O}(\mathrm{s})$ medicamento(s) teste e o referência devem apresentar as mesmas concentrações, formas farmacêuticas, características físicas e físico-químicas (10-11).

Para os testes de equivalência farmacêutica, é preferível a utilização de métodos analíticos presentes na monografia individual do medicamento, que se encontra na Farmacopeia Brasileira $5^{\circ}$ edição (12), mas quando não houver, pode-se fazer uso de outras farmacopeias que tenham autorização pela legislação vigente (13).

Um termo indispensável para a aprovação de medicamentos similares e genéricos é a bioequivalência, que nada mais é que uma comprovação entre produtos com a mesma quantidade de princípio ativo, mesma forma farmacêutica e biodisponibilidade, quando testados sob as mesmas condições. $\mathrm{O}$ que possibilita a conclusão da comparação da biodisponibilidade entre medicamento referência e teste é o critério de aceitação empregado pela bioequivalência. Esta conclusão determina, por meio de cálculo, se os medicamentos são ou não bioequivalentes, ou seja, se eles são intercambiáveis. Este é um critério adotado internacionalmente (12).

$\mathrm{O}$ que garante a intercambialidade entre medicamentos de referência e medicamentos genéricos é a Lei dos Genéricos, no 9.787 de 1999 (14). Desde então, cada vez mais a população brasileira vem buscando informações sobre os genéricos. Em 2018, o faturamento de genéricos se aproximou a 8,6 bilhões de reais, estimando-se um aumento de mais de $10 \%$ desse valor para o ano de 2019. No Brasil, os medicamentos genéricos apresentam $34,07 \%$ das vendas de medicamentos farmacêuticos (15).

Diante do exposto, este trabalho tem o objetivo de avaliar a equivalência farmacêutica de comprimidos de duas marcas de medicamentos genéricos (G1 e G2) e duas marcas de similares (S1 e S2) em relação ao medicamento referência $(\mathrm{R})$ de comprimido de furosemida $40 \mathrm{mg}$. 


\section{MATERIAIS E MÉTODO}

Materiais. As amostras utilizadas foram adquiridas em uma farmácia comercial da cidade de Mandaguaçu, PR, Brasil, e denominadas R para medicamento referência, G1 e G2 para medicamentos genéricos e S1 e S2 para medicamentos similares.

Foi utilizado um padrão secundário de furosemida 99,8\% (Vepakum L.18/FRS/042) obtido em farmácia de manipulação de Maringá (PR), nos testes de dissolução e doseamento. Como reagentes do teste de dissolução foram utilizados fosfato de potássio dibásico (Exxodo Científica) e hidróxido de potássio (Exxodo Cientifica).

Os equipamentos utilizados foram balanças analítica e semianalítica; aparelho desintegrador; espectrofotômetro de ultravioleta; friabilômetro; aparelho dissolutor; agitador magnético e pHmetro.

Determinação de peso. A determinação do peso médio foi realizada de acordo com metodologia preconizada na Farmacopeia Brasileira $5^{\text {a }}$ edição, 2010. Foram pesados 20 comprimidos de cada amostra em balança analítica, seguida da determinação da variação percentual do peso dos comprimidos em relação à média (12).

Doseamento. Para determinar a quantidade de substância ativa, foram pulverizados os $20 \mathrm{com}-$ primidos utilizados para a determinação do peso médio das amostras R, G1, G2, S1 e S2. Após preparo das amostras foi feita a leitura em espectrofotômetro de absorção no ultravioleta (Oceanoptics modelo UBS 2000+) com comprimento de onda de $271 \mathrm{~nm}$ e comparado com o padrão secundário de furosemida 99,8\% (Vepakum L.18/FRS/042). Foi utilizado hidróxido de sódio $0,1 \mathrm{M}$ para ajuste do zero e a quantidade de furosemida nos comprimidos foi calculada a partir das leituras obtidas (12).

Preparo do padrão. 0,2 $\mathrm{g}$ de furosemida foi dissolvido em $500 \mathrm{~mL}$ de hidróxido de sódio $0,1 \mathrm{M}$, e agitado em agitador magnético (Fisatom modelo 752A) por $10 \mathrm{~min}$. Após o tempo decorrido, a mistura foi filtrada e realizada a dissolução de $5 \mathrm{~mL}$ do filtrado em balão de $250 \mathrm{~mL}$ e o volume completado com hidróxido de sódio 0,1 M (12)

Preparo das amostras. Após pesagem de quantidade de pó equivalente a $0,2 \mathrm{~g}$ de furosemida das amostras R, G1, G2, S1 e S2, foram adicionados $500 \mathrm{~mL}$ de hidróxido de sódio $0,1 \mathrm{M}$ e homogeneizados em agitador magnético por 10 minutos. Após a homogeneização, foi transferida a quantidade de $5 \mathrm{~mL}$ para balão de $250 \mathrm{~mL}$ e o volume completado com o mesmo solvente. As análises foram realizadas em triplicata (12).

Teste de friabilidade. Para o teste de friabilidade foram pesados 10 comprimidos de cada marca (R, G1, G2, S1 e S2) em balança semianalítica (BEL modelo MARK 500). Os comprimidos foram colocados no friabilômetro (Nova Ética Mod. 300) e submetidos à ação do aparelho por 4 min. a 25 rotações/minuto, totalizando 100 rotações (12).

Após o teste, os comprimidos íntegros e livres de pó foram novamente pesados e a porcentagem de friabilidade calculada conforme preconizado pela Farmacopeia Brasileira $5^{\circ}$ edição (12).

Teste de desintegração. $O$ teste de desintegração foi realizado com 6 comprimidos de cada amostra, segundo os critérios descritos na Farmacopeia Brasileira (2010), utilizando água destilada em temperatura de $37 \pm 1{ }^{\circ} \mathrm{C}$ como líquido de imersão (12). Os comprimidos foram submetidos à ação do desintegrador (Nova Ética modelo 301-AC) por $30 \mathrm{~min}$. Decorrido este tempo foi analisado se havia a presença de comprimidos ou núcleos duros não desintegrados nos tubos do desintegrador.

Teste de dureza. Para este teste, foram utilizados 10 comprimidos de furosemida $40 \mathrm{mg}$, segundo os critérios descritos na Farmacopeia Brasileira (2010). A avaliação da dureza foi realizada em aparelho que aplica uma pressão sobre o comprimido até que ele se quebre, sendo a força medida em Newtons (N) (12).

Teste de dissolução. O teste de dissolução foi realizado conforme descrito na Farmacopeia Brasileira, 2010 (12). Foram utilizados $900 \mathrm{~mL}$ do meio de dissolução (tampão fosfato $\mathrm{pH} 5,8$ ), dissolutor (Nova Ética modelo 299), aparelhagem (pás; 50 $\mathrm{rpm})$, tempo (60 min.) e temperatura $\left(37 \pm 0,5^{\circ} \mathrm{C}\right)$. Decorrido o tempo especificado, uma alíquota ( 25 $\mathrm{mL}$ ) foi retirada de cada cuba, filtrada e diluída com tampão fosfato $\mathrm{pH} 5,8$ até a concentração teórica de $0,0008 \%(\mathrm{p} / \mathrm{v})$. As análises foram realizadas em triplicata. 
A leitura das amostras diluídas foi realizada em espectrofotômetro de absorbância no ultravioleta (Oceanoptics modelo UBS 2000+) a $271 \mathrm{~nm}$, para identificar a quantitativamente de fármaco dissolvido no meio de dissolução, e comparado com a leitura obtida para a solução padrão preparada na mesma concentração das amostras.

\section{Contagem do número total de microrganis-} mos mesófilos. O teste foi realizado em duplicata para todas as amostras. Foi utilizado o meio ágar caseína soja (TSA) para analisar o crescimento de microrganismos aeróbios totais; e o meio ágar Sabouraud dextrose para avaliar crescimento de fungos e leveduras (12).

Preparo das amostras. Foram utilizados tubos preenchidos com $5 \mathrm{~mL}$ de caldo caseína soja. O comprimido foi colocado no tubo e homogeneizado em vortex até sua completa desintegração.

Preparo das placas. Foi pipetado $1 \mathrm{~mL}$ da amostra dissolvida, em uma placa de petri e em seguida adicionado TSA ou ágar Sabouraud dextrose.
A amostra foi homogeneizada na placa segundo a técnica de Pour Plate. Depois do ágar solidificado, as placas foram mantidas em temperatura controlada de $32,5^{\circ} \mathrm{C} \pm 2,5^{\circ} \mathrm{C}$ durante 5 dias para as amostras com ágar caseína soja e $22,5^{\circ} \mathrm{C} \pm 2,5^{\circ} \mathrm{C}$ durante 7 dias para o ágar Sabouraud dextrose (12)

Ao final do teste foi observado se ocorreu crescimento ou não, de colônias de bactérias e fungos.

\section{RESULTADOS E DISCUSSÃO}

Determinação de peso. Um fator que influencia no peso de um comprimido são os excipientes e a quantidade de princípio ativo usado (16). Em um mesmo lote, podem ocorrer variações nos pesos dos comprimidos visto que as máquinas de compressão, uma vez não reguladas, podem comprimir quantidades erradas (17). Os valores obtidos na determinação de peso médio dos comprimidos de furosemida 40 mg (R, G1, G2, S1 e S2) estão na Tabela 1.

Tabela 1. Determinação de peso de comprimidos de furosemida $40 \mathrm{mg}$.

\begin{tabular}{|l|c|c|c|c|c|}
\hline & $\mathbf{R}$ & Amostra G1 & Amostra G2 & Amostra S1 & Amostra S2 \\
\hline Maior peso (g) & 0,168 & 0,168 & 0,204 & 0,171 & 0,156 \\
\hline Menor peso(g) & 0,156 & 0,156 & 0,190 & 0,159 & 0,140 \\
\hline Peso médio & $\mathbf{0 , 1 6 1}$ & $\mathbf{0 , 1 6 1}$ & $\mathbf{0 , 1 9 8}$ & $\mathbf{0 , 1 6 3}$ & $\mathbf{0 , 1 4 9}$ \\
\hline Mais 10\% & 0,1771 & 0,1771 & 0,2178 & 0,1793 & 0,1639 \\
\hline Menos 10\% & 0,1449 & 0,1449 & 0,1782 & 0,1467 & 0,1341 \\
\hline Desvio padrão & 0,0025 & 0,0037 & 0,0041 & 0,0029 & 0,0041 \\
\hline Resultado & Aprovado & Aprovado & Aprovado & Aprovado & Aprovado \\
\hline
\end{tabular}

Segundo os parâmetros estabelecidos pela Farmacopeia Brasileira (2010), para comprimidos com peso médio menor que $80 \mathrm{mg}$ o limite de variação permitido é de $\pm 10 \%$, podendo ter apenas duas unidades fora desse limite, mas nenhuma pode apresentar limite acima ou abaixo do dobro da porcentagem indicada (12). Sendo assim, todas as amostras testadas foram aprovadas neste parâmetro de qualidade.

Essas variações de peso entre comprimidos do mesmo lote ou entre comprimidos de diferentes marcas mostram uma relação de quantidade entre o ativo e os excipientes utilizados para a fa- bricação. Segundo um estudo de equivalência farmacêutica e perfil de dissolução comparativo de medicamentos contendo hidroclorotiazida, quanto mais pesado o medicamento, mais excipientes ele possui (18).

Teste de friabilidade. Teste mecânicos, como de dureza e friabilidade, têm o objetivo de determinar a integridade dos comprimidos. Com o teste de friabilidade é possível avaliar a resistência de um comprimido à ruptura que pode ocorrer devido a quedas e/ou fricção, durante todo o processo de produção, como embalagem, armazenamento, transporte etc. (16). Após o teste, os comprimidos 
não devem apresentar rachaduras, lascas ou danos que sejam visíveis (12). A amostra é considerada aprovada quando a perda de massa dos comprimidos não é superior a $1,5 \%$ (19). Na tabela 2 , estão os resultados do teste de friabilidade.

Visto que nenhum dos resultados apresentou perdas maiores que $1,5 \%$, todos os comprimidos foram aprovados no teste de acordo com o critério obtido na Farmacopeia Brasileira, 2010 (12). Apenas uma marca de comprimido de furosemida $40 \mathrm{mg}$ apresentou perda maior que o medicamento referência.
Em outro estudo de equivalência farmacêutica realizada com comprimidos de cloridrato de bupropiona em relação a similares, três marcas de comprimidos apresentaram valores de perda maior que o referência, no entanto foram aprovados, visto que nenhum teve perda maior que $1,5 \%$ (20).

Quatro das cinco amostras testadas apresentaram um baixo valor de perda de massa, como no teste de friabilidade realizado por Buzzi et al (21) em uma avaliação de qualidade de comprimidos de atenolol de marca similar e genérico, onde nenhuma das marcas apresentou perda superior a $0,4 \%$.

Tabela 2. Resultados obtidos no teste de friabilidade dos comprimidos de furosemida $40 \mathrm{mg}$.

\begin{tabular}{|l|c|c|c|c|c|}
\hline & $\mathbf{R}$ & Amostra G1 & Amostra G2 & Amostra S1 & Amostra S2 \\
\hline Peso inicial (g) & 3,237 & 3,231 & 3,971 & 3,278 & 2,958 \\
\hline Peso final (g) & 3,227 & 3,210 & 3,968 & 3,275 & 2,952 \\
\hline Perda (\%) & 0,31 & 0,65 & 0,08 & 0,1 & 0,21 \\
\hline Resultado & Aprovado & Aprovado & Aprovado & Aprovado & Aprovado \\
\hline
\end{tabular}

Teste de desintegração. Para estabelecer parâmetros de dissolução e absorção de um fármaco, o teste de desintegração é muito importante. Neste teste, usa-se o equipamento de desintegração, que tem o objetivo de desintegrar todo o comprimido em determinado tempo, temperatura e meio de imersão específicos. Ao acabar o tempo de desintegração, é preciso observar se todo o comprimido foi dissolvido ou mesmo se há massa sem núcleo palpável (16).

Os resultados do teste de desintegração podem ser observados na Tabela 3.

Tabela 3. Resultados obtidos no teste de desintegração dos comprimidos de furosemida $40 \mathrm{mg}$.

\begin{tabular}{|c|c|c|c|c|}
\hline \multicolumn{1}{|c|}{$\mathbf{R}$} & Amostra G1 & Amostra G2 & Amostra S1 & Amostra S2 \\
\hline $\begin{array}{c}\text { Todos os } 6 \text { compri- } \\
\text { midos desintegrados } \\
\text { em 23 segundos. }\end{array}$ & $\begin{array}{l}\text { Todos os } 6 \text { comprimi- } \\
\text { dos desintegrados em } \\
38 \text { segundos. }\end{array}$ & $\begin{array}{l}\text { Todos os } 6 \text { comprimi- } \\
\text { dos desintegrados em } \\
1 \text { minuto. }\end{array}$ & $\begin{array}{l}\text { Todos os } 6 \text { comprimi- } \\
\text { dos desintegrados em } 5\end{array}$ & $\begin{array}{l}\text { Todos os } 6 \text { comprimidos } \\
\text { desintegrados em } 2 \text { mi- } \\
\text { minutos e 28 segundos. }\end{array}$ \\
\hline Aprovados e 18 segundos. \\
\hline
\end{tabular}

Segundo a Farmacopeia Brasileira (2010), para comprimidos de furosemida $40 \mathrm{mg}$, o tempo de desintegração máximo permitido é de 30 minutos (12). Todas as marcas apresentaram uma desintegração mais rápida que a permitida, portanto, aprovadas no teste.

Em um estudo semelhante, feito por Bianchi e Souza (2012), em que foram realizados testes físicos de comprimidos de ibuprofeno, estes atenderam determinações de qualidade para peso mé- dio, friabilidade, dureza e desintegração, resultados que, quando comparados com este, mostram-se parecidos (22).

Em outro estudo de equivalência farmacêutica, realizado por Köhler e cols (2009), todos os 30 comprimidos de dipirona, referência, genéricos e similares foram desintegrados completamente em menos de 30 minutos, sendo aprovados nesse parâmetro (23). 
Teste de dureza. Este teste pode ser visto como teste de resistência mecânica, ou seja, é empregado uma força mecânica sobre os comprimidos até que os comprimidos se quebrem, anotando a força que foi utilizada até a ruptura, esta força é medida em Newton. O teste de dureza é somente informativo. Uma dureza elevada pode indicar uma desintegração mais lenta e assim afetar a absorção no organismo. Em relação aos resultados, a marca que necessitou de emprego de mais força para a ruptura foi a G2, com uma média de $5,71 \mathrm{~N}$. Todas as outras marcas necessitaram de força inferior a esta. Ajustes de pressão de compressão são realizados no meio de produção de comprimidos a partir deste teste (12).

Em uma avaliação da qualidade de comprimidos de hidroclorotiazida, de acordo com o coeficiente de variação, duas das três amostras testadas apresentaram valores elevados (24). Em outro estudo de controle da qualidade de comprimidos de captopril, foi feito o teste de dureza com 10 comprimidos e todos foram rompidos com força acima de $30 \mathrm{~N}$, mostrando que estavam nos valores que eram esperados (25).

Teste de contagem do número total de micro-organismos mesófilos. Um parâmetro essencial para a eficácia, aceitabilidade e principalmente segurança na fabricação de comprimidos industriais é a qualidade microbiológica. A reprovação nesta análise pode indicar falhas na produção, diante disso, as empresas utilizam do manual de boas práticas de fabricação, exigido pela Anvisa (26).

Somente são registradas, como resultado, placas de bactérias com crescimento inferior a 250 colônias e fungos com crescimento inferior a 50 colônias, segundo a Farmacopeia Brasileira, 2010 (12).

De acordo com parâmetros estabelecidos pela Farmacopeia Brasileira (2010) os limites aceitáveis para comprimidos são $10^{3} \mathrm{UFC} / \mathrm{mL}$ para a contagem das bactérias, e de $10^{2} \mathrm{UFC} / \mathrm{mL}$ para a contagem de fungos (12). Nenhuma amostra analisada apresentou crescimento de bactérias ou fungos, sendo aprovadas neste ensaio. As análises foram feitas em duplicata.

Teste de dissolução. A biodisponibilidade de um medicamento está diretamente relacionada com a dissolução que o comprimido apresenta. Os valo- res obtidos no teste de dissolução dos comprimidos de furosemida $40 \mathrm{mg}$ estão descritos na Tabela 4.

Tabela 4. Resultados obtidos no teste de dissolução dos comprimidos de furosemida.

\begin{tabular}{|c|c|c|}
\hline Amostras & $\begin{array}{l}\text { Substância ativa } \\
\text { dissolvida (\%) }\end{array}$ & (Média $\pm \mathrm{DP}$ ) \\
\hline R1 & 98,63 & \multirow{6}{*}{$95,19 \pm 5,13$} \\
\hline R2 & 98,63 & \\
\hline R3 & 89,56 & \\
\hline R4 & 96,37 & \\
\hline R5 & 87,98 & \\
\hline R6 & 100,00 & \\
\hline G1.1 & 100,00 & \multirow{6}{*}{$91,94 \pm 4,82$} \\
\hline $\mathrm{G} 1.2$ & 95,46 & \\
\hline G1.3 & 90,24 & \\
\hline G1.4 & 88,20 & \\
\hline G1.5 & 87,52 & \\
\hline G1.6 & 90,24 & \\
\hline $\mathrm{G} 2.1$ & 85,94 & \multirow{6}{*}{$92,92 \pm 6,22$} \\
\hline $\mathrm{G} 2.2$ & 98,86 & \\
\hline G2.3 & 86,39 & \\
\hline G2.4 & 100,22 & \\
\hline G2.5 & 90,47 & \\
\hline $\mathrm{G} 2.6$ & 95,69 & \\
\hline S1.1 & 101,36 & \multirow{6}{*}{$96,81 \pm 4,14$} \\
\hline S1.2 & 97,27 & \\
\hline S1.3 & 100,90 & \\
\hline S1.4 & 90,24 & \\
\hline S1.5 & 94,55 & \\
\hline S1.6 & 96,59 & \\
\hline S2.1 & 97,50 & \multirow{6}{*}{$95,49 \pm 8,12$} \\
\hline S2.2 & 92,06 & \\
\hline S2.3 & 111,11 & \\
\hline S2.4 & 89,34 & \\
\hline S2.5 & 90,92 & \\
\hline S2.6 & 92,06 & \\
\hline
\end{tabular}

Segundo a Farmacopeia Brasileira (2010), para comprimidos de furosemida $40 \mathrm{mg}$, a porcentagem da quantidade declarada do princípio ativo dissolvido é Q $(80 \%)+5 \%$, e o tempo do teste é de 30 minutos. Todas as amostras devem apresen- 
tar porcentagem de dissolução igual ou superior a $85 \%$, para que sejam consideradas aprovadas (12).

Diante dessas especificações, todas as amostras foram aprovadas, pois apresentaram uma dissolução maior que $85 \%$ em 30 minutos. Pelos resultados, a amostra S1 apresentou uma melhor homogeneidade na dissolução, sendo comprovada pelo seu desvio padrão. Os comprimidos da amostra R e os da amostra G1 apresentaram pequena diferença no tempo de dissolução. Os comprimidos da amostra S2, apresentaram os valores de dissolução mais discrepantes, com o maior desvio padrão.

A diferença individual entre comprimidos da mesma marca ou de marcas diferentes podem ter influência na disponibilidade no organismo, devido à relação entre a dissolução e a biodisponibilidade (7).

Segundo Brum e cols (2012), a eficácia clínica de um medicamento tem relação direta com a dissolução, visto que a velocidade do processo de dissolução é dependente da extensão da absorção do fármaco (27).

Um dos fatores que podem influenciar diretamente na dissolução de um medicamento são os excipientes utilizados na preparação. Para confirmar isso, Block e cols (2008) desenvolveram comprimidos de metformina $500 \mathrm{mg}$ com diferentes excipientes na fabricação testando-os com o de referên- cia. A menor eficiência de dissolução notada pelos autores foi a formulação contendo PVP K30® que é um polímero não iônico (28).

Doseamento. De acordo com a Farmacopeia Brasileira (2010), para comprimidos de furosemida $40 \mathrm{mg}$, o teor de furosemida pode variar de 90 a $110 \%$ do valor declarado no rótulo (12). Os resultados de teor obtidos para cada amostra estão descritos na Tabela 5.

Todas as cinco amostras foram aprovadas no teste de doseamento por estarem dentro do limite aceitável de 90 a $110 \%$ de substância ativa.

Em um estudo realizado por Bianchin e cols (2010), quanto à avaliação da qualidade de comprimidos de propranolol e enalapril distribuídos no sistema público de saúde em uma cidade do sul do Brasil, no teste de doseamento duas amostras de propranolol $40 \mathrm{mg}$ de um total de cinco foram reprovadas nesse quesito e uma apresentou teor limítrofe (29).

Em outro estudo semelhante realizado por Mariano e cols (2015), em testes de controle de qualidade físico-químico de comprimidos de dipirona sódica, de três amostras testadas no doseamento uma apresentou teor menor que o limite mínimo aceitável pela farmacopeia $(95 \%)$, sendo esta amostra reprovada (30).

Tabela 5. Valores obtidos no doseamento dos comprimidos de furosemida $40 \mathrm{mg}$.

\begin{tabular}{|c|c|c|c|c|c|}
\hline & R & G1 & G2 & S1 & S2 \\
\hline 1 & $99,51 \%$ & $95,80 \%$ & $106,26 \%$ & $105,10 \%$ & $105,52 \%$ \\
\hline 2 & $109,99 \%$ & $100,33 \%$ & $105,80 \%$ & $98,10 \%$ & $109,98 \%$ \\
\hline 3 & $104,27 \%$ & $104,02 \%$ & $109,24 \%$ & $107,35 \%$ & $104,17 \%$ \\
\hline Média & $104,59 \%$ & $100,05 \%$ & $107,10 \%$ & $103,52 \%$ & 6,58 \\
\hline Resvio padrão & 5,25 & 4,11 & 1,86 & 4,82 & Aprovado \\
\hline
\end{tabular}

\section{CONCLUSÃO}

De acordo com os testes realizados, os medicamentos testados (genéricos e similares) são equivalentes farmacêuticos ao medicamento referência quanto à variação de peso, friabilidade, desintegração, dureza, dissolução e doseamento. Em relação ao teste de contagem do número total de micro-organismos mesofilos, não houve constatação de crescimento fúngico ou bacteriano em qualquer uma das amostras analisadas. 
1. Linsbinsk LM, Musis CR, Machado SRP. Avaliação da equivalência farmacêutica de comprimidos de captopril. Rev. Bras. Farm. 2008;89(3):214-219.

2. OPAS. Organização Pan-Americana de Saúde. Dia Mundial da Hipertensão 2016. Disponível em: https://www. paho.org/bireme/index.php?option=com_content\& view=article $\&$ id=330: diamundial-da-hipertensao-2016 \&Itemid=183\&lang=en. Acesso em 20 de maio 2019.

3. Storpirtis S, Oliveira PG, Rodrigues D, Maranho D. Considerações biofarmacotécnicas relevantes na fabricação de medicamentos genéricos: fatores que afetam a dissolução e a absorção de fármacos. Rev. Bras. Cien. Farm. 1999;35(1): 1-16.

4. Granero GE, Longhi MR, Mora MJ, Junginger HE, Midha KK, Shah VP, Stavchansky S, Dressman JB, Barends DM. Biowaiver monographs for immediate release solid oral dosage forms: furosemide. J. Pharm. Sci. 2010;99(6):2544-2556. DOI: 10.1002/jps.22030

5. Silva P. Farmacologia. 6. ed. Rio de Janeiro: Guanabara. 1314, 2002

6. Veronez LL; Simões MJS. Análise da prescrição de medicamentos de pacientes hipertensos atendidos pelo SUS da rede municipal de saúde de Rincão - SP. Rev Ciência Farm Bas Aplic. 2008;29(1):45-51.

7. Menezes FG, Nascimento L, Willian J, Camargo RP, Greco KV, Santos AS. Perfil do uso de medicamentos para doenças cardiovasculares: análise de prescrições médicas em farmácia privada da região oeste da cidade de São Paulo. Saúde Colet. 2009;34(6):237-243.

8. ANVISA. Agência Nacional de Vigilância Sanitária. Furosemida Bula Profissional. Disponível em: http://www. anvisa.gov.br/datavisa/fila_bula/frmVisualizarBula.asp?pNuTransacao $=7629252015 \&$ pIdAnexo $=2825037$. Acesso em 20 de maio de 2019.

9. ANVISA. Agência Nacional de Vigilância Sanitária. Dispõe sobre as boas práticas de fabricação de medicamentos. Disponível em: http://bvsms.saude.gov.br/ bvs/saudelegis/anvisa/2010/res0017_16_04_2010.html. Acesso em 20 de maio de 2019.

10. Storpirtis S. A Equivalência Farmacêutica no Contexto da Intercambialidade entre Medicamentos Genéricos e de Referência: Bases Técnicas e Científicas. Infarma. 2004;16(9/10):51-55.

11. Pugens AM, Donaduzzi CM, Melo EB. Controle de Qualidade Total e Equivalência Farmacêutica de Três Apresentações de Captopril. Rev. Eletron. Farm. 2008; 5(1):32-45.

12. BRASIL. Farmacopeia Brasileira. 5a edição. 1:387-390. Disponível em: http://portal.anvisa.gov.br/documents/ $33832 / 260079 / 5 \% \mathrm{C} 2 \% \mathrm{AA}+\mathrm{edi} \% \mathrm{C} 3 \% \mathrm{~A} 7 \% \mathrm{C} 3 \%$ A 3 o+-+Volume+1/4c530f86-fe 83-4c4a-b907-6a96b 5c2d2fc. Acesso em 20 de maio 2019.
13. ANVISA. Agência Nacional da Vigilância Sanitária. Trata da admissibilidade das Farmacopeias estrangeiras. Disponível em: http://bvsms.saude.gov.br/bvs/saudelegis/anvisa/2009/res0037_06_07_2009.html. Acesso em 20 de maio de 2019.

14. BRASIL. Lei $\mathrm{n}^{\circ} 9787$ de 10 de fevereiro de 1999. Disponível em: http://www.planalto.gov.br/ccivil_03/LEIS/ L9787.htm. Acesso em 20 de maio de 2019.

15. PróGenéricos. Associação Brasileira De Indústrias De Medicamentos Genéricos. Mercado. Disponível em: http://progenericos.org.br/mercado. Acesso em 20 de maio de 2019.

16. Allen JR, Loyd V, Popovich NG, Ansel HC. Formas Farmacêuticas e Sistemas de Liberação de Fármacos. $9^{\circ}$ ed. Artmed Editora. 236-239, 2013.

17. Prista LN, Alves AC, Morgado R, Lobo JS. Tecnologia farmacêutica. 6. ed. Lisboa: Fundação Calouste Gulbenkian. 2013. 436-437.

18. Ribeiro CC, Andrade GMM, Couto RO. Estudos de equivalência farmacêutica e perfil de dissolução comparativo de medicamentos contendo Hidroclorotiazida. Infarma - Ciências Farm. 2018;30(1):5-13. DOI: 10.14450/2318-9312.v30.e1.a2018.pp5-13

19. BRASIL. Agência Nacional de Vigilância Sanitária. Resolução da Diretoria Colegiada (RDC) n ${ }^{\circ} 31$ de 11 de agosto de 2010.

20. Castro VL. Estudos de equivalência farmacêutica com comprimidos de Cloridrato de Bupropiona em medicamentos similar e referência. 2010. (dissertação) Universidade Católica de Góias.

21. Buzzi V. Avaliação da qualidade de comprimidos de atenolol: estudo comparativo entre medicamentos de referência, genérico e similar. Arq Ciências Saúde UNIPAR. 2006;10(3). DOI: 10.25110/arqsaude.v10i3.2006.2267

22. Bianchi C, Souza B. Monitoramento do processo de compressão de ibuprofeno utilizando controle estatístico. Rev Ciências Farm Bas Aplic. 2012;33(2): 255-265.

23. Kohler LF, Nascimento HD; Schwengber ELL, Bandeira ZMP, Pazin GV, Machado SRP. Avaliação Biofarmacotécnica e Perfil de Dissolução de Comprimidos de Dipirona: equivalência farmacêutica entre medicamentos de referência, genéricos e similares. Rev. Bras. Farm. 2009;90(4): 309-315.

24. Messa RV, Farinelli BCF, Menegati CFM. Avaliação na qualidade de comprimidos de hidroclorotiazida: medicamentos de referência, genérico e similar comercializados na cidade de Dourados - MS. Interbio. 2014;8(1): 73-76.

25. Peixoto MM, Santos Júnior AF, Santos CAA, Júnior EC. Avaliação da qualidade de comprimidos captopril dispensados em Feira de Santana - BA. Infarma. 2005;16(13/14): 69-73. 
26. Yamamoto CH, Andreoli Pinto TJ, Meurer VM, Carvalho AM, Rezende P. Controle de Qualidade Microbiológico de Produtos Farmacêuticos, Cosméticos e Fitoterápicos Produzidos na Zona da Mata, MG. Universidade Federal de Juiz de Fora - UFJF e Universidade de São Paulo USP.: 1-7, 2004.

27. Brum TF, Laporta LV, Pons Júnior FR, Gonçalves CA, Santos MR. Equivalência farmacêutica e estudo comparativo dos perfis de dissolução de medicamentos genéricos contendo paracetamol. Rev Ciências Farm Bas Aplicada. 2012;33(3):373-378.

28. Block LC, Schemling LO, Couto AG, Mourão SC, Bresolin TMB. Pharmaceutical Equivalence of Metformin Tablets with Various Binders. Rev. Ciênc. Farm. Básica Apl. 2008;29(1):29-35.
29. Bianchin MD, Blatt CR, Soares AS, Guerreiro ICK. Avaliação da qualidade de comprimidos de propranolol e enalapril distribuídos no sistema público de saúde em uma cidade do sul do Brasil. Cienc Saude Colet. 2012;17(2):491-498 DOI: 10.1590/ S1413-81232012000200022

30. Mariano MDC, Naves RHB, Freitas TA, Pinto MV, Fernandes CKC, Gonçalves Júnior AF, Souza SAO. Controle de Qualidade Físico-químico de Comprimidos de Dipirona Sódica Adquiridos em Drogarias de São Luis de Montes Belo (GO). Rev Fac Montes Belos (FMB). 2015;8(1):1-10. 